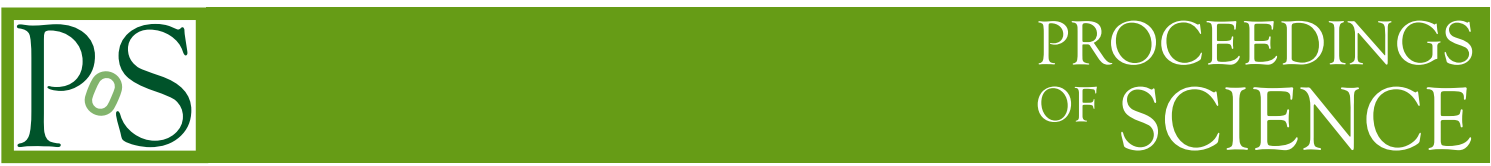

\title{
Quarkonia and Quarkonia-like spectroscopy at $\mathrm{LHCb}$
}

\section{Conor Fitzpatrick* European Organization for Nuclear Research (CERN), Geneva, Switzerland \\ E-mail: conor.fitzpatrickecern.ch}

\section{On behalf of the LHCb collaboration}

The LHCb experiment is optimised to study beauty and charm hadron decays at the Large Hadron Collider. The combination of precise tracking, particle identification and flexible software trigger make $\mathrm{LHCb}$ an ideal experiment with which to study exotic quarkonium states. These proceedings will discuss measurements of the $\mathrm{X}(3872)$ quantum numbers and a search for the decay $\mathrm{X}(4140) \rightarrow \mathrm{J} / \psi \phi$

XXI International Workshop on Deep-Inelastic Scattering and Related Subject -DIS2013, 22-26 April 2013

Marseilles, France

\footnotetext{
${ }^{*}$ Speaker.
} 


\section{Introduction}

In recent years a number of new quarkonia-like states have been studied at b-factories and hadron colliders. LHCb [1] is a dedicated beauty and charm experiment at the Large Hadron Collider (LHC). It is a single-arm spectrometer spectrometer design with polar-angle coverage of $15-300 \mathrm{mrad}$, and is ideally suited to measure the properties of these quarkonia-like states. In the 2011 running period of the LHC, LHCb collected the equivalent of $1 \mathrm{fb}^{-1}$ of integrated luminosity with proton-proton collisions at the center of mass energy $\sqrt{s}=7 \mathrm{TeV}$. With this dataset we have performed a measurement of the quantum numbers of the $\mathrm{X}(3872) \rightarrow \mathrm{J} / \psi \pi^{+} \pi^{-}$in the decay $\mathrm{B}^{ \pm} \rightarrow \mathrm{X}(3872) \mathrm{K}^{ \pm}$, and a search for the state $\mathrm{X}(4140) \rightarrow \mathrm{J} / \psi \phi$ in the decay $\mathrm{B}^{ \pm} \rightarrow \mathrm{X}(4140) \mathrm{K}^{ \pm}$.

\section{2. $\mathrm{X}(3872) \rightarrow \mathrm{J} / \psi \pi^{+} \pi^{-}$}

In the decade since its first observation by Belle [2], the $\mathrm{X}(3872)$ has been confirmed by a number of experiments $[3,4,5]$. The structure of the $\mathrm{X}(3872)$ still remains unclear however. $B A B A R$ and Belle observed the decay $\mathrm{X}(3872) \rightarrow \mathrm{J} / \psi \gamma$, indicating that the C-parity of the $\mathrm{X}(3872)$ is positive $[3,4]$. The angular analysis was performed by $\mathrm{CDF}$ excluded all quantum numbers except for $J^{P C}=2^{-+}, 1^{++}[5]$. The $J^{P C}=2^{-+}$configuration would indicate a more conventional charmonium state, although the measured $\mathrm{X}(3872)$ mass is not particularly consistent with that of the nearest charmonium state, the $\eta_{\mathrm{c} 2}\left(1^{1} \mathrm{D}_{2}\right)$. More exotic states have been proposed for the $\mathrm{X}(3872)$, such as a tetraquark [6] or loosely-bound $\mathrm{D}^{*} \overline{\mathrm{D}}^{0}$ molecule [7]. LHCb has performed a five-dimensional angular analysis of $\mathrm{X}(3872)$ candidates produced in the decay of $\mathrm{B}^{ \pm}$mesons to the $\mathrm{J} / \psi \pi^{+} \pi^{-} \mathrm{K}^{ \pm}$ final state [8] in order to determine the $\mathrm{X}(3872)$ quantum numbers. The kinematics of this decay chain fully determines the polarisation of the $\mathrm{X}(3872)$. Candidates are selected by requiring a high $p_{\mathrm{T}} \mathrm{J} / \psi \rightarrow \mu^{-} \mu^{+}$displaced from the primary vertex and with a good vertex fit $\chi^{2}$ consistent with the mass of the $\mathrm{J} / \psi$. A $\mathrm{K}^{+} \pi^{-} \pi^{+}$comnination is then selected and which also forms a good vertex and is subject to particle ID requirements. The $\mathrm{B}^{+}$candidate vertex fit must be of high quality, and a minimum decay time requirement of $0.25 \mathrm{ps}$ is imposed to reject backgrounds from the primary vertex. To further reduce backgrounds a likelihood ratio is constructed using as inputs $x_{i}$ several discriminating variables. The probability density functions (PDFs) of these distributions are obtained from $\mathrm{J} / \psi \pi^{+} \pi^{-} \mathrm{K}^{ \pm}$sidebands for the background and simulated candidates of the topologically identical $\mathrm{B}^{ \pm} \rightarrow \psi(2 \mathrm{~S}) \mathrm{K}^{ \pm}$mode as signal. We require $-2 \sum_{i=1}^{4} \ln \left[\mathscr{L}_{\text {sig }}\left(x_{i}\right) / \mathscr{L}_{\text {bkg }}\left(x_{i}\right)\right]<1.0$ which is $94 \%$ efficient on $\mathrm{X}(3872)$ signal candidates. Around 38000 candidates are selected within $2 \sigma$ of the $\mathrm{B}^{ \pm}$peak in the $M\left(\mathrm{~J} / \psi \pi^{+} \pi^{-} \mathrm{K}^{ \pm}\right)$distribution, with a signal purity of $89 \%$. The mass difference $\Delta M=M\left(\mathrm{~J} / \psi \pi^{+} \pi^{-}\right)-M(\mathrm{~J} / \psi)$ distribution is shown in Figure 1. Fits to the $\psi(2 \mathrm{~S})$ and $\mathrm{X}(3872)$ signals are shown in the insets, in which a Crystal Ball function [9] with symmetric tails is used to describe the signal lineshape and a straight line is used to describe the background. The fit to the $\psi(2 \mathrm{~S})$ mass window has no fixed parameters and is found to have a $\Delta M$ resolution of $\sigma_{\Delta M}=3.99 \pm 0.05 \mathrm{MeV}$ and a yield of $5642 \pm 76$ signal candidates. The fit to the $\mathrm{X}(3872)$ mass window uses signal tail parameters fixed to those determined from the $\psi(2 \mathrm{~S})$, yielding $313 \pm 26$ signal candidates with a $\Delta M$ resolution of $\sigma_{\Delta M}=5.5 \pm 0.5 \mathrm{MeV}$. The $\psi(2 \mathrm{~S})$ signal purity within $\pm 2.5 \sigma_{\Delta M}$ is $68 \%$. In order to distinguish between the $J^{P C}=1^{++}, 2^{-+}$quantum numbers a likelihood ratio, $t$, is constructed from PDFs corresponding to the two possible states. These PDFs 


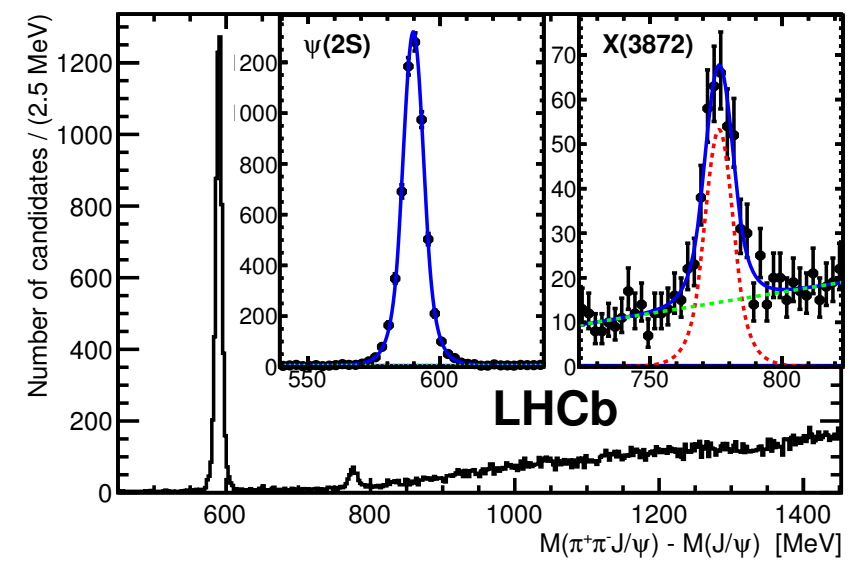

Figure 1: $\Delta M$ distribution for $\mathrm{J} / \psi \pi^{+} \pi^{-} \mathrm{K}^{ \pm}$candidates. Fits to the $\psi(2 \mathrm{~S})$ and $\mathrm{X}(3872)$ signals are overlaid. The solid blue, dashed red and dotted green lines represent the total fit, signal component and background component, respectively.

exploit the full power of the angular correlations in the decay. Each PDF is the product of the matrix element and reconstruction efficiency. The matrix element encodes angular information in the helicity basis. The helicity couplings are expressed in terms of the $L S$ couplings [10] where $L$ is the orbital angular momentum between the $\pi \pi$ system and the $\mathrm{J} / \psi$ meson and $S$ is the sum of their spins. In the $J^{P C}=1^{++}$case $L=0$ and $S=1$ : The angular distribution is completely determined without free parameters. For the $J^{P C}=2^{-+}$case $L=1$ which implies $S=1$ or 2 . As both $L S$ combinations are possible this implies two free parameters which are chosen to be the real and imaginary parts of the complex parameter $\alpha$, defined by the relative contribution of the amplitudes. In this way $t=-2 \ln \left[\mathscr{L}\left(2^{-+}\right) / \mathscr{L}\left(1^{++}\right)\right]$may be determined from fitting to the data twice, once with and once without the free parameters $\mathfrak{R e}(\alpha), \mathfrak{I m}(\alpha)$. Numerical simulations of both hypotheses are used to compare to the measured value of $t$ in data, and are found to produce approximately Gaussian distributions in $t$ which are well separated. The measured value of $t$ in data is found to be +99 , which favors the $1^{++}$hypothesis. The value of $\alpha$ which minimises $-2 \ln \left[\mathscr{L}\left(2^{-+}\right)\right]$is found to be $\mathfrak{R} \mathfrak{e}(\alpha)=0.671 \pm 0.046, \mathfrak{I m}(\alpha)=0.280 \pm 0.046$ which is both consistent with that reported by Belle and with the value measured from a large sample of simulated $1^{++}$candidates. The comparison between data and simulated experiments of both $J^{P C}$ hypotheses is shown in Figure 2. The distribution of $t$ values obtained from simulated $J^{P C}=2^{-+}$experiments is roughly consistent with a Gaussian distribution. Based on the mean and r.m.s of this distribution, rejection of the $J^{P C}=2^{-+}$hypothesis is achieved at $8.4 \sigma$ significance. The discriminating power of the angular correlations cannot be overemphasized. Figure 3 shows the distribution of background-subtracted $X(3872)$ candidates as a function of $\cos \left(\theta_{X}\right)$ where $\theta_{X}$ is the angle made by the $\pi^{+} \pi^{-}$combination momentum vector with respect to the $\mathrm{X}(3872)$ momentum vector. This distribution is shown for all candidates (top) and for candidates after requiring that the angle made by the $\pi^{-}$with respect to the $\pi^{+} \pi^{-}$combination momentum vector $\theta_{\pi \pi}$ satisfies $\left|\cos \left(\theta_{\pi \pi}\right)\right|>0.6$ (bottom). Overlaid are the distributions obtained in simulated $J^{P C}=1^{++}$and $J^{P C}=2^{-+}$events. It can be seen that there is little difference between the two hypotheses when integrating over all 


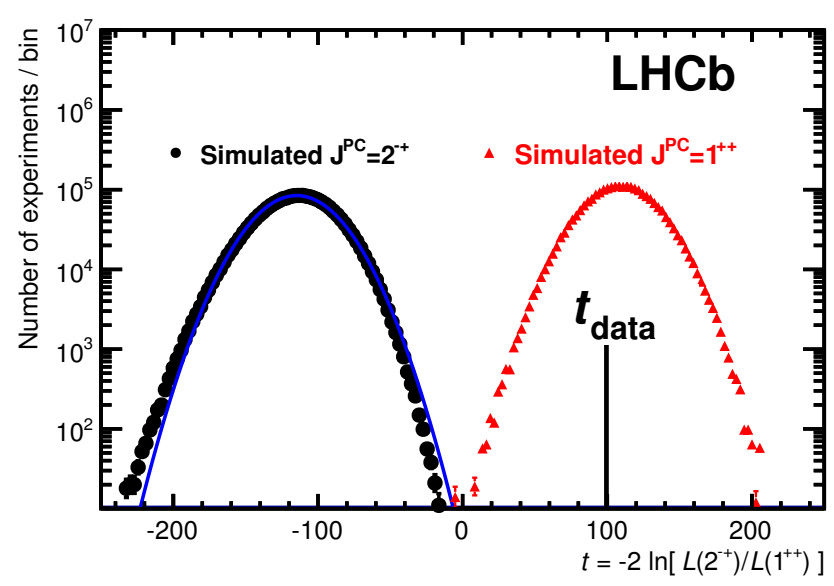

Figure 2: Distribution of the test statistic $t$ for simulated experiments with $J^{P C}=2^{-+}$(black circles) and $J^{P C}=1^{++}$(red triangles). The result obtained from data is shown as the black line at +99 . A Gaussian fit to the $2^{-+}$distribution is overlaid (blue line).

$\left|\cos \left(\theta_{\pi \pi}\right)\right|$, but large differences are observed when the correlations are taken into account.

\section{3. $\mathrm{X}(4140) \rightarrow \mathrm{J} / \psi \phi$}

CDF observed a narrow structure decaying to $\mathrm{J} / \psi \phi$ in the decay $\mathrm{B}^{ \pm} \rightarrow \mathrm{J} / \psi \phi \mathrm{K}^{ \pm}$, finding $3.8 \sigma$ significance [11], later updated to $>5 \sigma$ [12]. In the updated measurement they also observed an additional structure at $4274 \mathrm{MeV}(>3 \sigma)$. The narrow nature of the $\mathrm{X}(4140)$ is unusual for a conventional charmonium state, leading to speculation that it may be exotic [13, 14, 15]. Belle performed a search for the $\mathrm{X}(4140)$ in $\gamma \gamma \rightarrow \mathrm{J} / \psi \phi$ decays, which was unable to confirm the CDF result, disfavoring a molecular interpretation [16]. LHCb has performed a search for a narrow resonance in the same channel as $\mathrm{CDF}$, using the equivalent of $0.37 \mathrm{fb}^{-1}$ of integrated luminosity with proton-proton collisions at center of mass energy $7 \mathrm{TeV}$ [17]. $\mathrm{B}^{ \pm} \rightarrow \mathrm{J} / \psi \mathrm{K}^{+} \mathrm{K}^{-} \mathrm{K}^{ \pm}$candidates are selected in a very similar manner to that of the $\mathrm{X}(3872)$ analysis. A likelihood ratio is constructed using simulated $\mathrm{B}^{ \pm} \rightarrow \mathrm{J} / \psi \mathrm{K}^{+} \mathrm{K}^{-} \mathrm{K}^{ \pm}$to determine signal distributions and data sidebands to model background. The value of the likelihood ratio which maximises the figure of merit $S / \sqrt{S+B}$ for signal, $S$ and background extrapolated into the signal region, $B$ is used to determine the selection. A fit is performed to the invariant mass of selected $\mathrm{J} / \psi \mathrm{K}^{+} \mathrm{K}^{-} \mathrm{K}^{ \pm}$candidates after requiring that at least one $\mathrm{K}^{+} \mathrm{K}^{-}$combination is within $\pm 15 \mathrm{MeV}$ of the $\phi$ mass, shown in Figure 4 . The distribution is modelled using a Gaussian signal and quadratic background component. The fit finds $346 \pm 20 \mathrm{~B}^{ \pm}$candidates with a resolution of $5.2 \pm 0.3 \mathrm{MeV}$. Using candidates within $2.5 \sigma$ of the $\mathrm{B}^{ \pm}$mass peak a fit is also performed to the $\mathrm{K}^{+} \mathrm{K}^{-}$distribution, shown in the same figure. Here both combinations of $\mathrm{K}^{+} \mathrm{K}^{-}$are shown. The resonance is modelled using a relativistic P-wave Breit-Wigner lineshape fixed to the PDG value of the $\phi$ width while the non-resonant component is described by a two-body phase space model. Both models are convolved with a Gaussian resolution function. An additional component with the same resonant shape is included to account for peaking $\phi$ background determined from $\mathrm{B}^{ \pm}$sidebands. The background subtracted $\mathrm{B}^{ \pm} \rightarrow \mathrm{J} / \psi \phi \mathrm{K}^{ \pm}$yield is determined to be $382 \pm 22$ candidates. To search for the $\mathrm{X}(4140)$, only events within $\pm 15 \mathrm{MeV}$ of 


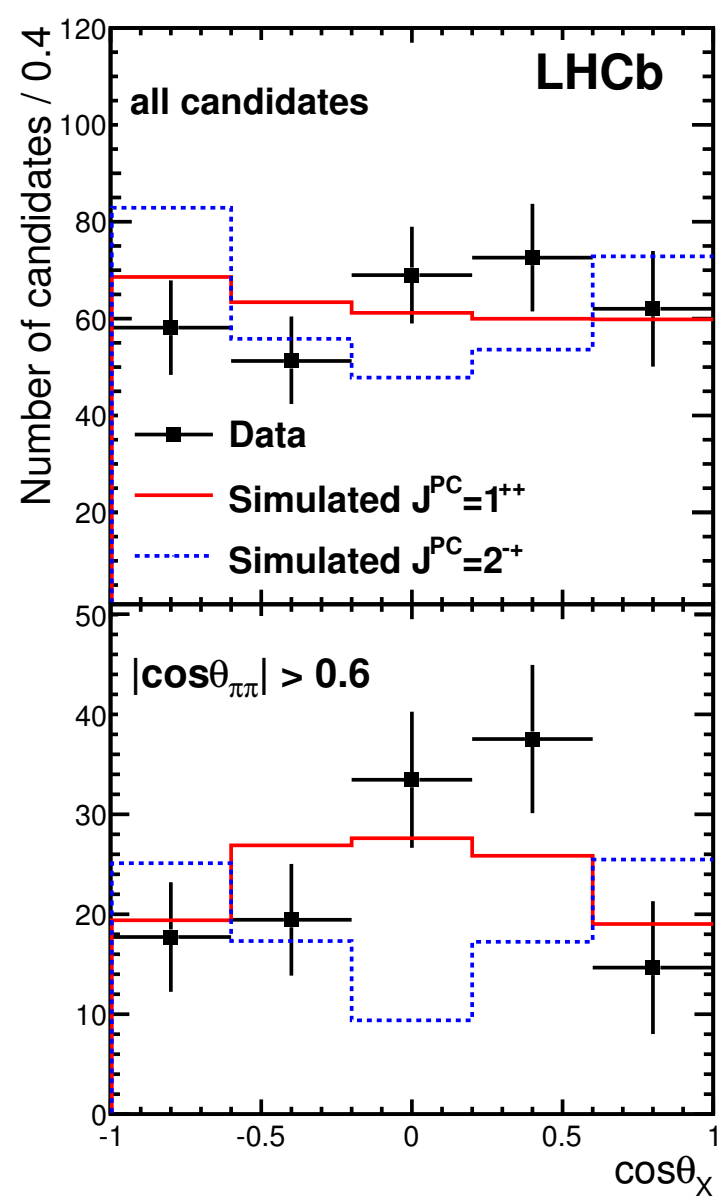

Figure 3: Distributions of one of the five helicity angles used to determine the quantum numbers of the $\mathrm{X}(3872)$. Top: $\cos \left(\theta_{X}\right)$ for all background subtracted $\mathrm{X}(3872)$ candidates. Bottom: $\cos \left(\theta_{X}\right)$ only for candidates with $\left|\cos \left(\theta_{\pi \pi}\right)\right|>0.6$. Overlaid are the distributions for simulated $J^{P C}=2^{-+}$(dashed lines) and $J^{P C}=1^{++}$candidates (solid lines). The improvement in discrminating power when taking into account the angular correlations is clear.

the $\phi$ mass are used, which is $85 \%$ efficient with respect to the total yield. The $M(\mathrm{~J} / \psi \phi)-M(\mathrm{~J} / \psi)$ invariant mass distribution of these candidates is shown in Figure 5. In Figure 5 (a) we fit to the mass difference distribution using the same model used in the CDF analysis [12] consisting of a spin-zero Breit Wigner signal component for each of the $\mathrm{X}(4140)$ and $\mathrm{X}(4274)$ whose mass and widths are fixed to the central values obtained by the CDF collaboration. The background consists of a three-body phase space distribution. Both signal and background shapes are convolved with the detector resolution, determined to be $1.5 \pm 0.1 \mathrm{MeV}$ from fully simulated $\mathrm{B}^{ \pm} \rightarrow \mathrm{X}(4140) \mathrm{K}^{ \pm}$ candidates. We obtain an $\mathrm{X}(4140)$ signal yield of $6.9 \pm 4.9$ events, and an $\mathrm{X}(4274)$ yield of $3.4_{-3.4}^{+6.5}$ events. Removing the $\mathrm{X}(4274)$ component from the fit does not affect the $\mathrm{X}(4140)$ yield. In Figure 5 (b) we use a different background parameterisation to explore the sensitivity of our results to the assumed background shape. Here we use a quadratic function multiplied by an efficiencycorrected three-body phase-space factor to impose the kinematic threshold. In this instance the fitted value of the $\mathrm{X}(4140)$ yield is 0.6 events with a positive error of 7.1 events. The $\mathrm{X}(4274)$ 

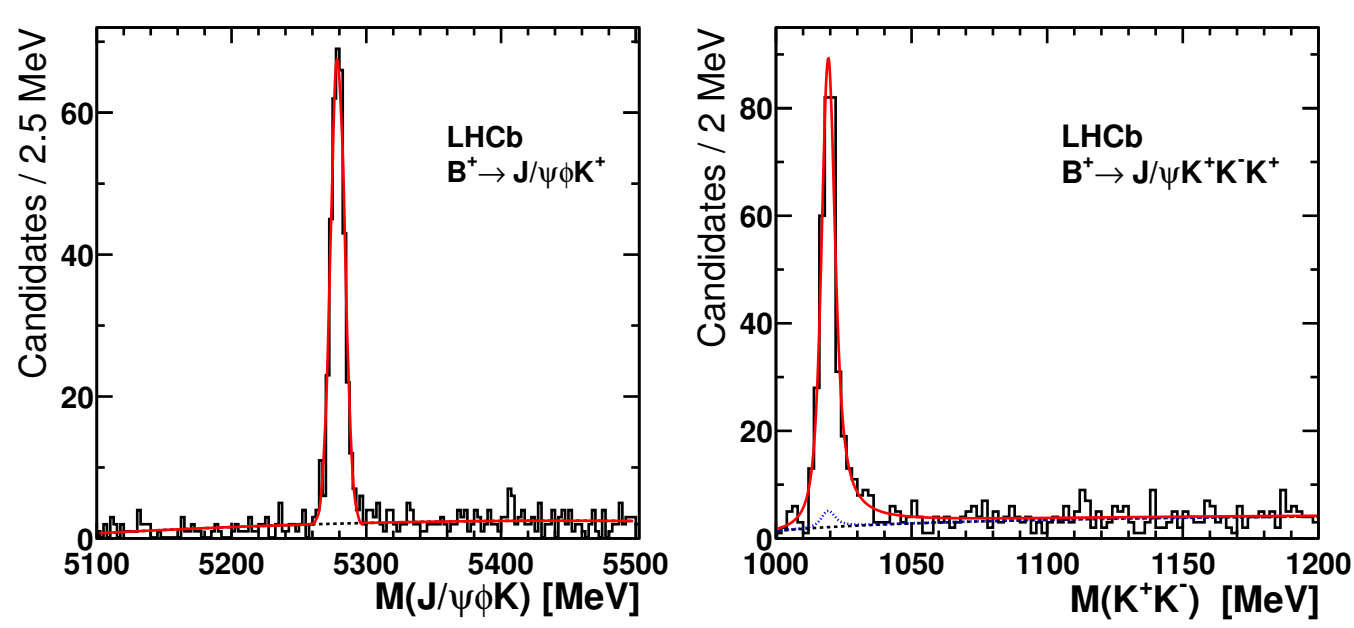

Figure 4: Left: Invariant mass distribution of $\mathrm{B}^{ \pm} \rightarrow \mathrm{J} / \psi \phi \mathrm{K}^{ \pm}$candidates after requiring that at least one $\mathrm{K}^{+} \mathrm{K}^{-}$combination is within $\pm 15 \mathrm{MeV}$ of the $\phi$ mass. A fit is overlaid in red, consisting of a Gaussian signal component and a quadratic background (dashed line). Right: Invariant mass distribution of $\mathrm{K}^{+} \mathrm{K}^{-}$ combinations from candidates within the $2.5 \sigma$ region around the $\mathrm{B}^{ \pm}$mass peak. A fit is overlaid in red, using a P-wave relativistic Breit-Wigner to model the signal component. The dashed line indicates the twobody phase-space contribution. The dotted line indicates the genuine $\phi$ background contribution estimated from a fit to the $\mathrm{B}^{ \pm}$sidebands.

yield is determined to be 0 with a positive error of 10 events. We determine a relative efficiency between simulated $\mathrm{B}^{ \pm} \rightarrow \mathrm{X}(4140) \mathrm{K}^{ \pm}$and $\mathrm{B}^{ \pm} \rightarrow \mathrm{J} / \psi \phi \mathrm{K}^{ \pm}$candidates distributed according to the phase space model to be $0.62 \pm 0.02$. Using our $\mathrm{B}^{ \pm} \rightarrow \mathrm{J} / \psi \phi \mathrm{K}^{ \pm}$signal yield multiplied by this efficiency ratio and the CDF value of the branching fraction [12], we predict that we should have observed $35 \pm 9 \pm 6$ events where the first uncertainty is statistical from the CDF data and the second includes both CDF and LHCb systematic uncertainties. Taking the statistical and systematic uncertainties from both experiments into account we find that our result disagrees with the CDF observation by $2.4 \sigma(2.7 \sigma)$ when using the first (second) background shape. As we do not find evidence of either narrow structure, we set an upper limit on the branching fractions:

$$
\begin{aligned}
\mathscr{B}\left(\mathrm{B}^{ \pm} \rightarrow \mathrm{X}(4140) \mathrm{K}^{ \pm}\right) \times \mathscr{B}(\mathrm{X}(4140) \rightarrow \mathrm{J} / \psi \phi) / \mathscr{B}\left(\mathrm{B}^{ \pm} \rightarrow \mathrm{J} / \psi \phi \mathrm{K}^{ \pm}\right) & <0.07 @ 90 \% \mathrm{CL} \\
\mathscr{B}\left(\mathrm{B}^{ \pm} \rightarrow \mathrm{X}(4274) \mathrm{K}^{ \pm}\right) \times \mathscr{B}(\mathrm{X}(4274) \rightarrow \mathrm{J} / \psi \phi) / \mathscr{B}\left(\mathrm{B}^{ \pm} \rightarrow \mathrm{J} / \psi \phi \mathrm{K}^{ \pm}\right) & <0.08 @ 90 \% \mathrm{CL}
\end{aligned}
$$

\section{Conclusions}

The LHCb experiment is competitive in the field of exotic quarkonia. These proceedings have presented a measurement of the quantum numbers of the $\mathrm{X}(3872)$ decaying to $\mathrm{J} / \psi \pi^{+} \pi^{-}$in the $\mathrm{B}^{ \pm} \rightarrow \mathrm{J} / \psi \pi^{+} \pi^{-} \mathrm{K}^{ \pm}$channel with $1 \mathrm{fb}^{-1}$. We find the $\mathrm{X}(3872)$ to be consistent with $J^{P C}=1^{++}$, favoring an exotic interpretation and rejecting the $2^{-+}$configuration at $>8$ sigma. With $0.37 \mathrm{fb}^{-1}$ we find no evidence of either the $\mathrm{X}(4140)$ or the $\mathrm{X}(4274)$ states decaying to $\mathrm{J} / \psi \phi$ in the $\mathrm{B}^{ \pm} \rightarrow \mathrm{J} / \psi \phi \mathrm{K}^{ \pm}$channel. This is in disagreement at the $2.4 \sigma$ level with the observation reported by the CDF collaboration. 


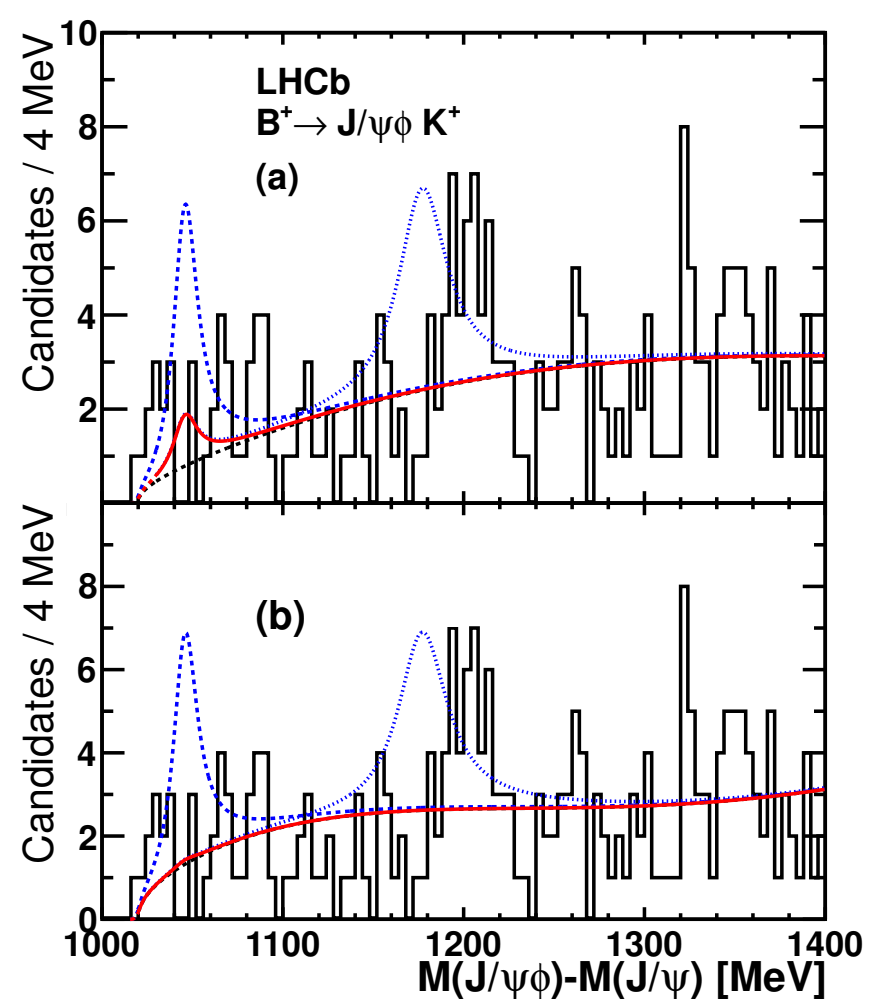

Figure 5: Distribution of the mass difference $M(\mathrm{~J} / \psi \phi)-M(\mathrm{~J} / \psi)$ for selected $\mathrm{B}^{ \pm} \rightarrow \mathrm{J} / \psi \phi \mathrm{K}^{ \pm}$candidates. Top: The distribution is modelled using a spin-zero Breit Wigner and three-body phase space background convolved with the detector resolution. Bottom: The background component is replaced with a quadratic function. In both cases the expected yields based on the CDF measurement are shown for the X(4140) and $\mathrm{X}(4274)$ as dashed lines.

\section{References}

[1] LHCb Collaboration, The LHCb Detector at the LHC, JINST 3 (2008) S08005.

[2] Belle Collaboration, S. Choi et. al., Observation of a narrow charmonium - like state in exclusive B+-> K+- pi+ pi-J/psi decays, Phys.Rev.Lett. 91 (2003) 262001, [hep-ex/ 0309032 ].

[3] BaBar Collaboration, B. Aubert et. al., Search for $B^{+} \rightarrow X(3872) K^{+}, X_{3872} \rightarrow J / \psi \gamma$, Phys.Rev. D74 (2006) 071101, [hep-ex/0607050].

[4] Belle Collaboration, V. Bhardwaj et. al., Observation of $X(3872) \rightarrow J / \psi \gamma$ and search for $X(3872) \rightarrow \psi^{\prime} \gamma$ in B decays, Phys.Rev.Lett. 107 (2011) 091803, [arXiv:1105.0177].

[5] CDF Collaboration, A. Abulencia et. al., Analysis of the quantum numbers $J^{P C}$ of the $X(3872)$, Phys.Rev.Lett. 98 (2007) 132002, [hep-ex/ 0612053 ].

[6] L. Maiani, F. Piccinini, A. Polosa, and V. Riquer, Diquark-antidiquarks with hidden or open charm and the nature of X(3872), Phys.Rev. D71 (2005) 014028, [hep-ph/ 0412098$].$

[7] N. A. Tornqvist, Isospin breaking of the narrow charmonium state of Belle at 3872-MeV as a deuson, Phys.Lett. B590 (2004) 209-215, [hep-ph / 0402237$].$

[8] LHCb Collaboration, R. Aaij et. al., Determination of the X(3872) meson quantum numbers, Phys. Rev. Lett. 110, 222001 (2013) [arXiv: 1302 . 6269]. 
[9] T. Skwarnicki, A study of the radiative CASCADE transitions between the Upsilon-Prime and Upsilon resonances, .

[10] N. Mangiafave, Measurements of Charmonia Production and a Study of the X(3872) at LHCb, .

[11] CDF Collaboration, T. Aaltonen et. al., Evidence for a Narrow Near-Threshold Structure in the $J / \psi \phi$ Mass Spectrum in $B^{+} \rightarrow J / \psi \phi K^{+}$Decays, Phys.Rev.Lett. 102 (2009) 242002, [arXiv:0903.2229].

[12] CDF Collaboration, T. Aaltonen et. al., Observation of the $Y(4140)$ structure in the $J / \psi \phi$ Mass Spectrum in $B^{ \pm} \rightarrow J / \psi \phi K$ decays, arXiv:1101.6058.

[13] F. Stancu, Can Y(4140) be a c anti-c s anti-s tetraquark?, J.Phys. G37 (2010) 075017, [arXiv:0906.2485].

[14] Possible Molecular States of $D^{*}(s)$ anti- $D^{*}(s)$ System and Y(4140), Eur.Phys.J. C64 (2009) 297-308, [arXiv:0904.1782].

[15] N. Drenska, R. Faccini, and A. Polosa, Exotic Hadrons with Hidden Charm and Strangeness, Phys.Rev. D79 (2009) 077502, [arXiv: 0902 .2803].

[16] Belle Collaboration, C. Shen et. al., Evidence for a new resonance and search for the $Y(4140)$ in the gamma gamma $\rightarrow$ phi J/psi process, Phys.Rev.Lett. 104 (2010) 112004, [arXiv: 0912.2383 ].

[17] LHCb Collaboration, R. Aaij et. al., Search for the $X(4140)$ state in $B^{+} \rightarrow J / \psi \phi K^{+}$decays, Phys.Rev. D85 (2012) 091103, [arXiv: 1202 . 5087]. 